\title{
Management of Congenital Deafness of the Syndrome Branchio-Oto- Renal (BOR): A Case Report
}

\author{
Mohamed Ali Gliti ${ }^{1,3 *}$, Khaoula Karim ${ }^{1,3}$, Bencheikh Razika ${ }^{2,3}$, Benbouzid Mohamed Anas ${ }^{2,3}$, Leila Essakalli Houssyni ${ }^{2,3}$
}

${ }^{1}$ Resident Physician In Otorhinolaryngology, Department of Otorhinolaryngology, Head and Neck Surgery, Ibn Sina University Hospital, Rabat, Morocco

${ }^{2}$ Professor of Otorhinolaryngology, Department of Otorhinolaryngology, Head and Neck Surgery, Ibn Sina University Hospital, Rabat, Morocco

${ }^{3}$ Faculty of Medicine and Pharmacy of Rabat, Mohammed V University, Rabat, Morocco

*Corresponding author: Mohamed Ali Gliti

Abstract

Objective: Describe the different therapeutic modalities of Branchio-oto-renal (BOR) Syndrome. Material and method: We report the case of a 7-year-old boy who presented with a Branchio-oto-renal syndrome (BOR). Clinical Case: T.S, a 7-year-old child presented from the age of 3 years, a progressive, bilateral deafness, with a preserved language. The otological examination found implanted low ears, bilateral prehelic fistulas, and left seromucosal otitis CT scan showed bilateral vestibulo-labyrinthine malformation confirmed by the MRI. Cochlear implant surgery on the right ear was performed. Conclusion: The therapeutic principle is based on the precociousness of the rehabilitation of a quality auditory canal, which remains a factor of good prognosis of bilateral deafness of the child reached of the syndrome of BOR. A quality ear canal is defined by an ability to perceive and understand speech by the ear canal alone. Severe or deep bilateral deafness is the subject of hearing rehabilitation by hearing aid or cochlear implant, speech therapy and educational support.

Keywords: Branchio-oto-renal (BOR) Syndrome, congenital deafness, hearing defects, cochlear implant.

Copyright $(\mathcal{C} 2020$ The Author(s): This is an open-access article distributed under the terms of the Creative Commons Attribution 4.0 International License (CC BY-NC 4.0) which permits unrestricted use, distribution, and reproduction in any medium for non-commercial use provided the original author and source are credited.

\section{INTRODUCTON}

Branchio-oto-renal syndrome (BOR) combines deafness, multiple gill fistulas and renal malformation. Its prevalence is estimated at 1 / 40,000. Renal malformations can be major (agenesis or major hypoplasia) and sometimes lead to termination of pregnancy. Lesser deformities will be diagnosed by a renal ultrasound which must be requested in the face of deafness suggestive of BOR: deafness is accompanied by malformations of the outer ear (poorly hemmed ears, ear aplasia, enchondromas, stenosis of the ear canals), the middle ear (there is a transmissive component to the audiogram) and the inner ear (various cochleovestibular malformations). We generally find bilateral preheliceal fistulas and fistulas of the second branchial cleft with associated cartilage residues suggestive. In practice, in the event of perceptual or mixed deafness associated with a gill fistula or malformations of the outer ear, it is desirable to do a renal ultrasound [1-19].

Three genes have been located and two identified, EYA1 and SIX1. The EYA1 gene can also be responsible for a branchio-otological syndrome, very close to BOR but without kidney damage [20-25].

\section{Case Report}

This is T.S, a 7-year-old child (Figure-1), 4th in a family of four. Without notion of neonatal suffering, hospitalization in intensive care, or taking ototoxic drugs in his history. No inbreeding among parents. The mother, a brother, an older sister, 3 maternal uncles, two maternal cousins, present more or less the same symptomatology as the child. 


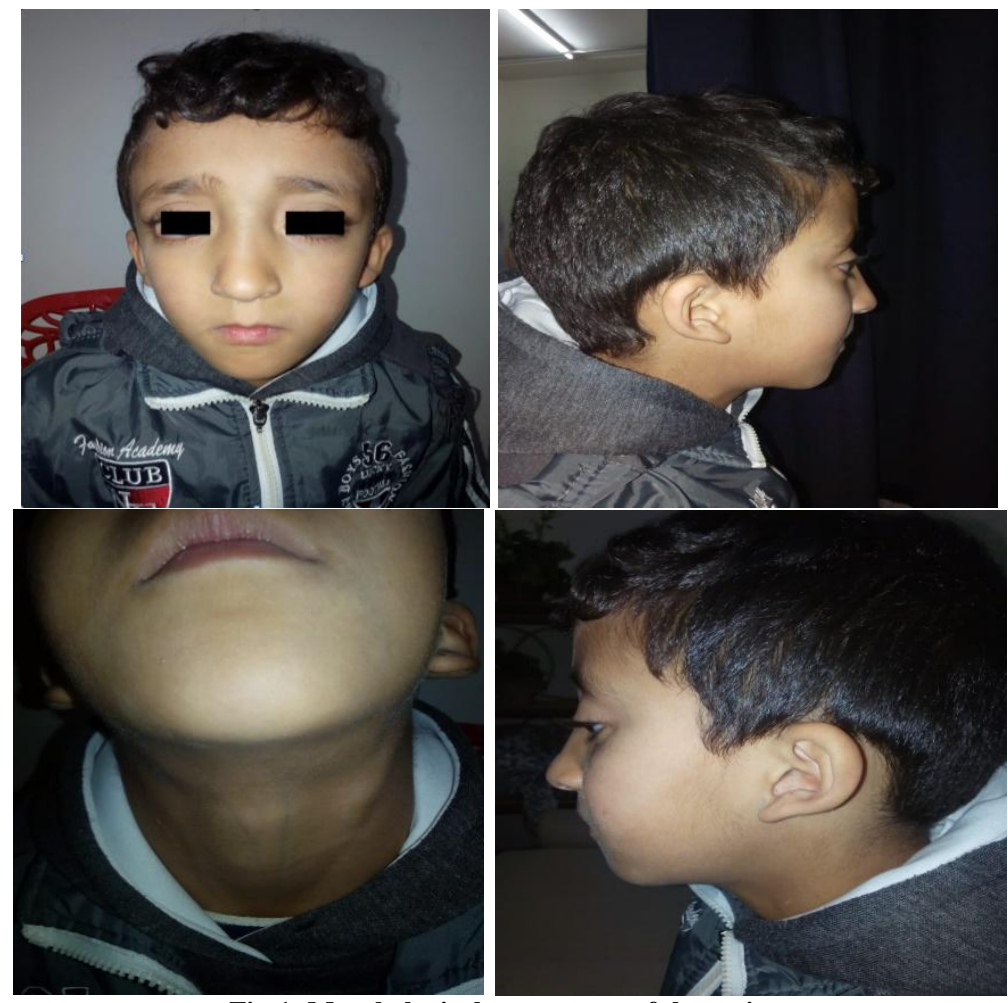

Fig-1: Morphological appearance of the patient

The patient presented from the age of 3 years, a progressive, bilateral deafness, with a preserved language. The otological examination found implanted low ears, bilateral prehelic fistulas, and left seromucosal otitis, the child reacts to the otoscopic aspiration. The cervical examination found a second bilateral cleft fistula. Audiological explorations have shown on the auditory evoked potential a bilateral cophosis (Figure2).

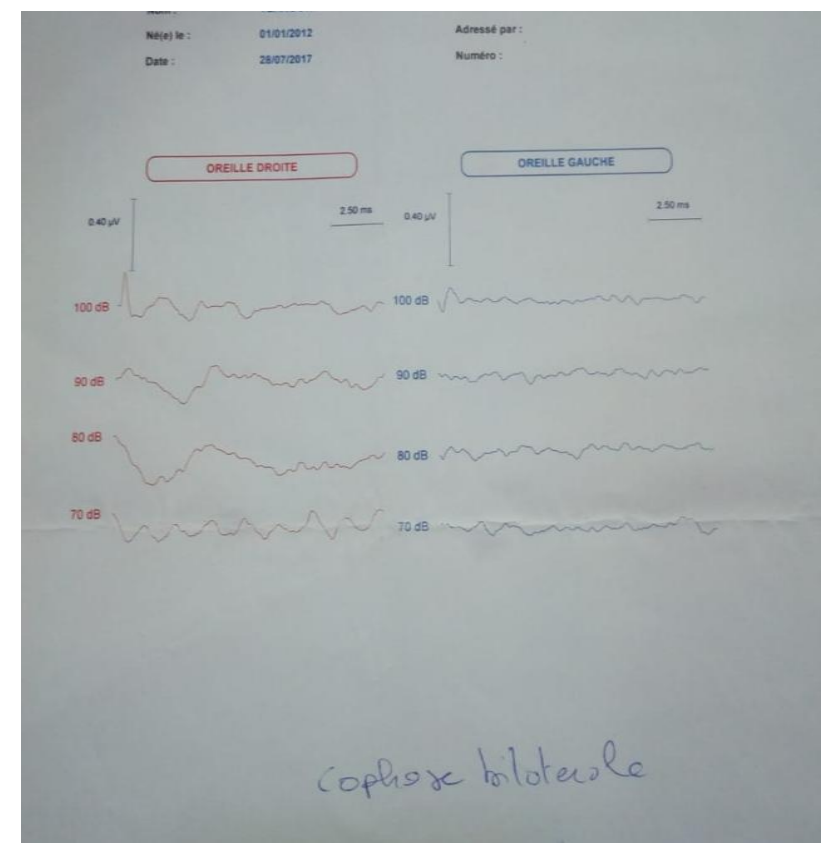

Fig-2: Evoked auditory potential showing bilateral cophosis in children

CT scan of of the petrous bones showed bilateral chronic otitis media with mastoiditis, and bilateral vestibulo-labyrinthine malformation in the form of bilateral vestibular ectasia, dilation of the median part of the canals, circular seedlings on both sides, with dilation of the aqueduct of the right vestibule (Figure-3). Kidney ultrasound is normal. No explorations done to family members. 

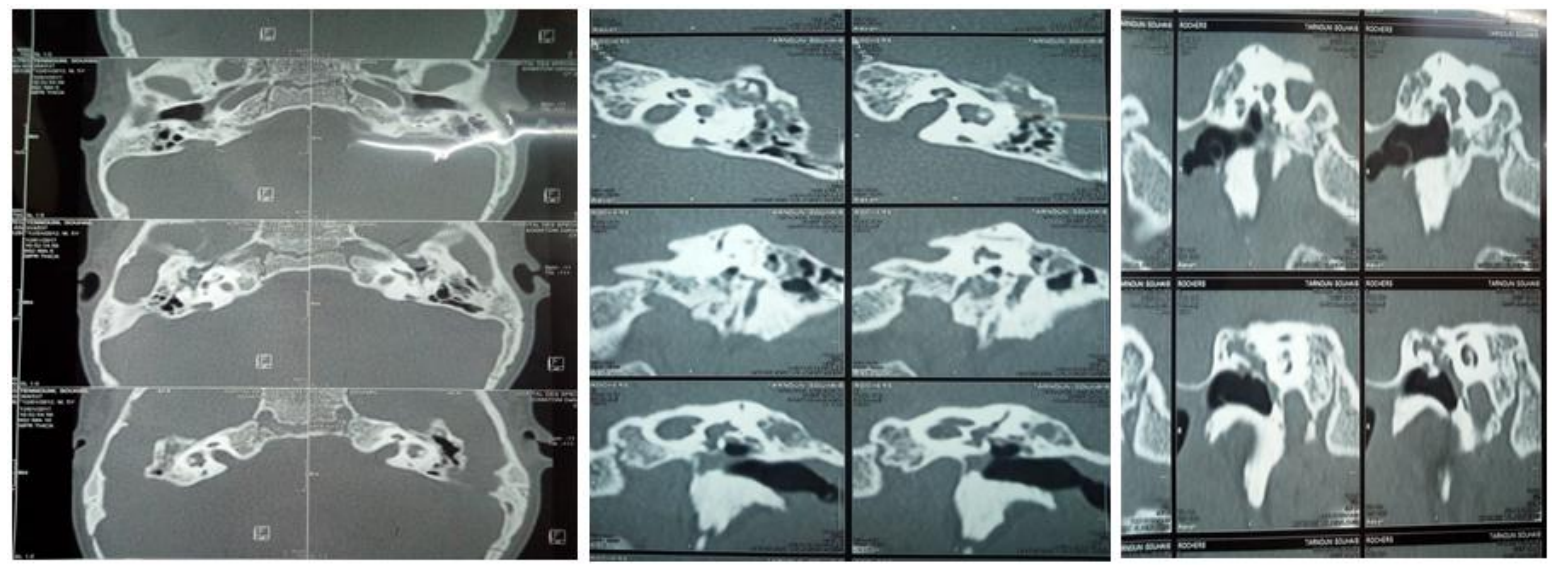

Fig-3: CT images with gadolinium injection in axial sections showing the different malformations of the inner ear on both sides

The MRI performed confirmed the malformations visible on the scanner, but these patients

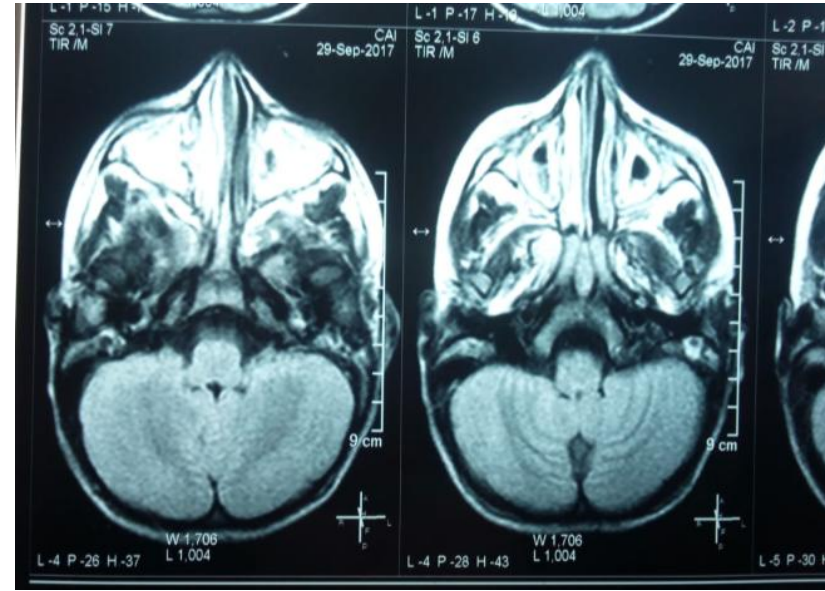

Fig-4: MRI images in axial sections with gadolonium injection in did not contraindicate the performance of cochlear implant surgery on the right ear (Figure-4).

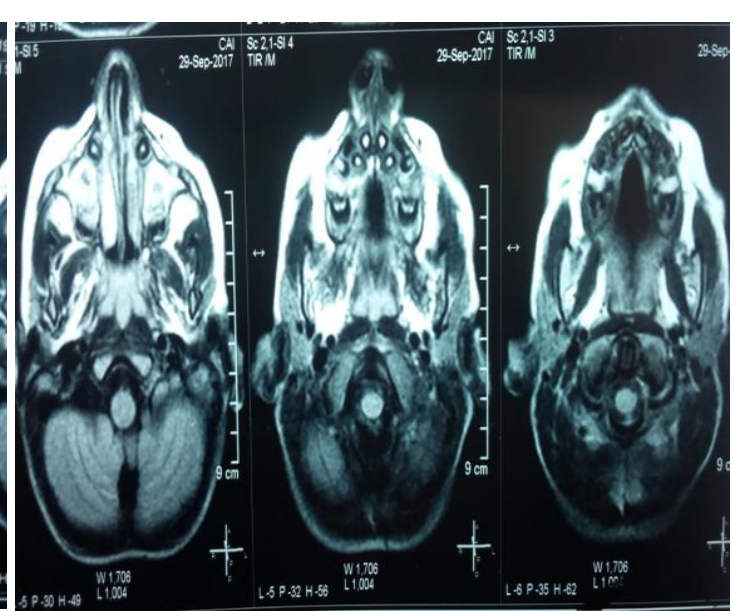

\section{DISCUSSION}

The branchio-oto-renal syndrome (BOR syndrome) has a complete penetration (any carrier of an anomaly in one of the genes involved presents clinical signs; no "generation jump"). Hearing loss is the most constant of the anomalies and affects $90 \%$ of patients. It can be transmission (30\%), neurosensory $(20 \%)$ or mixed in 50\% of cases. Deafness is severe in a third of patients and progressive in a quarter [26-36].

The treatment of BOR syndrome deafness, like all congenital deafness, imperatively concerns two aspects: on the one hand, on hearing loss itself, on the other hand on its consequences on learning. Early rehabilitation is essential, therefore, rehabilitation must be organized as soon as reliable and consistent arguments are obtained for permanent deafness: Early acoustic evoked potentials (PEAP) or Auditory Steady State Response (ASSR), and behavioral audiometry [37-49].

Hearing rehabilitation must start from the first months of life in cases where the deafness is severe or profound bilaterally. Speech therapy is essential.
Indeed, in most cases, compensation for hearing loss is not enough to make up for the delay in language development or learning essential for good schooling [50-56].

In infants and children, only hearing aid hearing aids are used, due to the small size and continuous growth of the external ear canal, which in most cases is malformed. In-ear devices are reserved for the adolescent, in case of refusal of another solution [57-62].

When deafness is progressive, having initially allowed access to oral language, the cochlear implant must be proposed as soon as speech perception without lip reading becomes less than $50 \%$ at $65 \mathrm{~dB}$ with two hearing aids [63-65].

Cochlear implantation remains a real surgical challenge given the different anatomical malformations found in this syndrome. Bilateral implantation is recommended because it allows better localization of sounds and better perception in noisy environments than unilateral implantation $[66,67]$. 


\section{CONCLUSION}

The significant advances in early hearing screening, imaging, and cochlear implantation have dramatically changed the future of children with BOR syndrome, allowing for better consideration of the impact of conductive hearing loss.

Once the diagnosis has been established and rehabilitation has started, the frequency of associated disorders and the potential development of deafness justify careful monitoring throughout schooling and multidisciplinary care in many cases.

\section{REFERENCES}

1. Bureau international d'audiophonologie. Recommanda- tion $02 / 1$ bis. Classification des déficiences auditives. www.biap.org/biapfrancais.htm.

2. Yoshinaga-Itano C. From screening to early identification and intervention: discovering predictors to successful outcomes for children with significant hearing loss. J Deaf Stud Deaf Educ 2003;8:11-30.

3. Kral A, Sharma A. Developmental neuroplasticity after cochlear implantation. Trends Neurosci 2012;35:111-22.

4. Haute Autorité de santé. Recommandations de bonne pra- tique. Surdité de l'enfant : accompagnement des familles et suivi de l'enfant de 0 à 6 ans, hors accompagnement scolaire. Argumentaire. Décembre 2009. p. 86. www.recomed.fr/sites/ default/files/node\%3Atitle/surdite de lenfant - 0 a 6 ans - argumentaire.pdf.

5. Golz A, Netzer A, Westerman ST, Westerman LM, Gilbert DA, Joachims HZ, et al. Reading performance in children with otitis media. Otolaryngol Head Neck Surg 2005; 132:495-9.

6. Wake M, Tobin S, Cone-Wesson B, Dahl HH, Gillam L, McCormick L, Poulakis Z, Rickards FW, Saunders K, Ukoumunne OC, Williams J. Slight/mild sensorineural hearing loss in children. Pediatrics. 2006 Nov 1;118(5):1842-51.

7. Hall AJ, Maw AR, Steer CD. Developmental outcomes in early compared with delayed surgery for glue ear up to age 7 years: a randomised controlled trial. Clin Otolaryngol 2009;34:12-20.

8. Fulcher A, Purcell AA, Baker E, Munro N. Listen up: chil- dren with early identified hearing loss achieve age-appropriate speech/language outcomes by 3 years-of-age. Int J Pediatr Otorhinolaryngol 2012;76:1785-94.

9. Haute Autorité de santé. Évaluation du dépistage néonatal systématique de la surdité permanente bilatérale, 2007 www.hassante.fr/portail/upload/docs/application/p $\mathrm{df} /$ rapportevaluation $\mathrm{du}$ depistage neonatal systematique de la surdite permanente bilaterale.pdf.
10. Lutman ME, Grandori F. Screening for neonatal hearing defects. European consensus statement. Eur J Pediatr, 1999;158: 95-6.

11. Boishardy AD, Lenoir FM, Brami P, Kapella M, Obstoy MF, Amstutz-Montadert I, Lerosey Y. Expérience du dépistage auditif néo-natal systématique dans le département de l'Eure: A propos de 10835 nouveau-nés. InAnnales d'Otolaryngologie et de Chirurgie Cervico-faciale 2005 Nov 1 (Vol. 122, No. 5, pp. 223-230). Elsevier Masson.

12. Kolski C, Le Driant B, Lorenzo P, Vandromme L, Strunski V. Early hearing screening: what is the best strategy? Int J Pediatr Otorhinolaryngol, 2007; 7:1055-60.

13. Dauman R, Roussey M, Belot V, Denoyelle F, Roman S, Gavilan-Cellié I, Ruzza-Surroca I, Calmels MN, Lina-Granade G, Houssin E, Charlemagne A. Screening to detect permanent childhood hearing impairment in neonates transferred from the newborn nursery. International journal of pediatric otorhinolaryngology. 2009 Mar 1;73(3):457-65.

14. Langagne T, Lévêque $\mathrm{M}$, Schmidt $\mathrm{P}$, Chays A. Universal newborn hearing screening in the Champagne-Ardenne region: a 4-year follow-up after early diagnosis of hearing impairment. Int $\mathbf{J}$ Pediatr Otorhinolaryngol, 2010;74:1164-70.

15. Arrêté du 23 avril 2012 relatif à l'organisation du dépistage de la surdité permanente néonatale. Journal Officiel de la Répu- blique fran,caise $n^{\circ}$ 0105 du 4 mai 2012, page 7915 . Texte $n^{\circ} 48$.

16. Garabédian N. Neonatal hearing-loss screening is on the way to becoming systematic in France. Eur Ann Otorhinolaryng Head Neck Surg, 2015;132:313.

17. Doyle KJ, Kong YY, Strobel K, Dallaire P, Ray RM. Neonatal middle ear effusion predicts chronic otitis media with effusion. Otol Neurotol, 2004;25:318-22.

18. Chen JL. Newborn hearing screening may predict Eus- tachian tube dysfunction. Int $\mathrm{J}$ Pediatr Otorhinolaryngol, 2015;79:2099-103.

19. Fortnum HM, Summerfield AQ, Marshall DH, Davis AC, Bamford JM. Prevalence of permanent childhood hearing impairment in the United Kingdom and implications for universal neonatal hearing screening: questionnaire based ascertainment study. Br Med J, 2001;323:536-40.

20. Joint Committee on Infant Hearing. Year 2000 position statement: principles and guidelines for early hearing detection and intervention programs. Pediatrics, 2000;106: 798-817.

21. Foulon I, Vleurinck L, Kerkhofs K, Gordts F. Hearing configu- ration in children with cCMV infection and proposal of a flow chart for hearing evaluation. Int J Audiol, 2015;54:714-9.

22. Société de pathologie infectieuse de langue fran caise. Prise en charge des méningites bactériennes aiguës commu- nautaires (à 
l'exclusion du nouveau-né). 17e conférence de consensus en thérapeutique anti-infectieuse, 2008. www.chu-besancon.fr/meningite/2008-

Meningites-court.pdf.

23. Peleva E, Emami N, Alzahrani M, Bezdjian A, Gurberg J, Carret AS, Daniel SJ. Incidence of platinum- induced ototoxicity in pediatric patients in Quebec. Pediatric blood \& cancer. 2014 Nov;61(11):2012-7.

24. Société fran caise de pédiatrie, avec le soutien de la Direc- tion générale de la santé. Dépistage des troubles de l'audition chez l'enfant, 2009. www.sante.gouv.fr/IMG/pdf/Depistage des troubles de 1 audition chez 1 enfant.pdf.

25. Bureau international d'audiophonologie. Recommandation 24/1 : développement du langage chez l'enfant de 0 à 3 ans. www.biap.org/biapfrancais.htm.

26. Gouma P, Mallis A, Daniilidis V, Gouveris H, Armenakis N, Naxa- kis S. Behavioral trends in young children with conductive hearing loss: a case-control study. Eur Arch Otorhinolaryngol 2011;268:63-6.

27. Ayral M, Baylan MY, Kinis V, Bez Y, Bakir S, Ozbay M, Yorgancilar E, Gun R, Topcu I. Evaluation of hyperactivity, attention deficit, and impulsivity before and after adenoidectomy/adenotonsillectomy surgery. Journal of Craniofacial Surgery. 2013 May 1;24(3):731-4.

28. Lieu JE. Speech-language and educational consequences of uni- lateral hearing loss in children. Arch Otolaryngol Head Neck Surg 2004;130:524-30.

29. Société fran caise d'audiologie. Le Guide de bonnes pra- tiques en audiométrie de l'enfant, $2009 . \quad$ www.sfaudiologie. fr/Drupal/sites/default/files/GBPAE.pdf.

30. Delaroche M. Audiométrie comportementale du très jeune enfant. Bruxelles: DeBoeck Université; 2001.

31. Audio4, test vocal fréquentiel rapide pour les enfants de 4 ans. orl.pediatrique@chu-tours.fr.

32. Estève-Fraysse MJ. Les potentiels évoqués auditifs précoces. In: Deguine O, Darrouzet V, editors. Électrophysiologie en ORL. Paris: Société Fran ,caise d'ORL; 2008;35-51.

33. Korczak P, Smart J, Delgado R, Strobel TM, Bradford C. Auditory steady-state responses. Journal Am Acad Audiol, 2012;23:146-70.

34. Fran ,cois M, Teissier N, Barthod G, Nasra Y. Sedation for children 2 to 5 years of age undergoing auditory brainstem response and auditory steady stateresponses recordings. Int $\mathbf{J}$ Audiol, 2012;51:282-6.

35. Précis d'audiométrie ou livre sur résultats audio+tympano+PEA.

36. Truy E, Ionescu E, Lina-Granade G, Butnaru C, Thai-Van H, Furminieux V, Collet L. Neuropathie auditive: clinique et revue de la littérature: A propos de 7 observations. InAnnales d'Otolaryngologie et de Chirurgie Cervico-faciale 2005 Dec 1 (Vol. 122, No. 6, pp. 303-314). Elsevier Masson.

37. Deltenre P, Collette JL. La neuropathie auditive/désynchronisation auditive. Cah Audit 2007;20:13-76, et 2008;21:6-79.

38. Berlin CI, Hood LJ, Morlet T, Wilensky D, Li L, Mattingly KR, Taylor-Jeanfreau J, Keats BJ, John PS, Montgomery E, Shallop JK. Multi-site diagnosis and management of 260 patients with auditory neuropathy/dys-synchrony (auditory neuropathy spectrum disorder*). International journal of audiology. 2010 Jan 1;49(1):30-43.

39. Manchaiah VK, Zhao F, Danesh AA, Duprey R. The genetic basis of auditory neuropathy spectrum disorder (ANSD). Int J Pediatr Otorhinolaryngol, 2011;75:151-8.

40. Demanez L, Dony-Closon B, Lhonneux-Ledoux F, Demanez JP. Central auditory processing assessment: a French-speaking battery. Acta Otorhinolaryngol Belg, 2003;57:275-90.

41. Collet L, Roge B, Descouens D, Moron P, Duverdy F, Urgell H. Objective auditory dysfunction in infantile autism. Lancet, 1993;342:923-4.

42. Rotenberg BW, Makhija M, Papsin BC. Conversion disorder in a child presenting as sudden sensorineural hearing loss. Int J Pediatr Otorhinolaryngol, 2005;69:1261-4.

43. Drouillard M, Francois M, Noel Petroff N, Majer J, Perrot C, Tabarino F. Circonstances et moyens $\mathrm{du}$ diagnostic des pseudo-hypoacousies. Ann Fr Oto-Rhino- Laryngol, 2012;129(suppl4:A73). http://www.em-consulte. com/en/article/751494 COR0005.

44. [44] L'aplasie majeure d'oreille. Plaquette d'information du centre de référence. http://www.malformationsorl.aphp. fr/IMG/pdf/Aplasi maj.pdf.

45. Noël-Petroff N. Cent syndromes ORL avec surdité. Paris: EDP Sciences; 2014.

46. Veillon F, Casselman JW. Imagerie de l'oreille et de l'os tem- poral, Volume 5 : Pédiatrie. Paris: Lavoisier; 2013.

47. Song JJ, Choi HG, Oh SH, Chang SO, Kim CS, Lee JH. Unilateral sensorineural hearing loss in children: the importance of tem- poral bone computed tomography and audiometric follow-up. Otol Neurotol, 2009;30:604-8.

48. Loundon N, Marlin S. La fœtopathie à cytomégalovirus. Action Connaissances Formation Surdité, Paris, 2007. http:// www.acfos.org/wpcontent/uploads/base doc/sciences tech niques/cytomegalovirus revue22.pdf.

49. Blanchard M, Thierry B, Marlin S, Denoyelle F. Aspects géné- tiques de la surdité. Arch Pediatr, 2012;19:886-9.

50. Calendrier des vaccinations et recommandations vaccinales 2015. Ministère des Affaires Sociales, 
de la Santé et des Droits des Femmes, Paris, 2015; 22-23.

51. ACFOS. Troubles de l'équilibre chez l'enfant. Associa- tion Connaissances Formation Surdité, Paris, $2012 . \quad$ www. acfos.org/publication/autresp/troublesequilibre juin2012.pdf.

52. Société fran caise d'ORL et de chirurgie cervicofaciale. Place de l'aérateur transtympanique dans le traitement de l'otite séro-muqueuse bilatérale. Réfé- rentiels professionnels de bonne pratique, Paris, 2008. www.orlfrance.org/article.php?id=42.

53. Gordon K, Henkin Y, Kral A. Asymmetric hearing during develop- ment: the aural preference syndrome and treatment options. Pediatrics, 2015; 136:141-53.

54. Garabédian EN, Bobin S, Monteil JP, Triglia JM. ORL de l'enfant. Paris: Médecine Sciences Flammarion; 2006.

55. Fuchsmann C, Tringali S, Disant F, Buiret G, Dubreuil C, Froehlich P, Truy E. Hearing rehabilitation in congenital aural atresia using the bone-anchored hearing aid: audiological and satisfaction results. Acta oto-laryngologica. 2010 Dec 1;130(12):1343-51.

56. Verhaert N, Fuchsmann C, Tringali S, LinaGranade G, Truy E. Strategies of active middle ear implants for hearing reha- bilitation in congenital aural atresia. Otol Neurotol, 2011;32:639-45.

57. Kesser BW, Krook K, Gray LC. Impact of unilateral conductive hearing loss due to aural atresia on academic performance in children. Laryngoscope, 2013;123:2270-5.

58. Banga R, Doshi J, Child A, Pendleton E, Reid A, McDermott AL. Bone-anchored hearing devices in children with unilateral conductive hearing loss: a patient-carer perspective. Ann Otol Rhinol Laryngol, 2013;122:582-7.

59. Franzoni M. Quelle place pour l'orthophoniste dans la prise en charge précoce de l'enfant sourd?
Association Connaissances Formation Surdité, Paris, 2006. www.acfos.org/sedocumenter/base doc/depistage/placeortho revue17.pdf.

60. Code de la sécurité sociale. Articles L622-3-6 et L321-1-3.

61. Bureau international d'audiophonologie. Recommandation 06/11: appareillage auditif chez l'enfant dans la première année. www.biap.org/fr/recommandations/66-ct-6-aidesaud itives-aca/15-recommandation-biap-0611appareillage-auditif -chez-lenfant-dans-lapremiere-annee.

62. INPES. La surdité de l'enfant. Guide pratique à l'usage des parents. Paris, 2005. www.inpes.sante.fr/ CFESBases/catalogue/pdf/884.pdf.

63. Boons T, Brokx JP, Dhooge I, Frijns JH, Peeraer L, Vermeulen A, Wouters J, Van Wieringen A. Predictors of spoken language development following pediatric cochlear implantation. Ear and hearing. 2012 Sep 1;33(5):617-39.

64. Fran, cois M, Boukhris M, Noel-Petroff N. Scolarité de l'enfant sourd et bénéfice d'un diagnostic précoce. Eur Ann Otorhino- laryng Head Neck Dis, 2015;132:251-5.

65. Haute Autorité de santé. Le traitement de la surdité par implants cochléaires ou du tronc cérébral Fiche de bon usage des technologies de santé (BUTS), Actualisation Janvier 2012. www.hassante.fr/portail/upload/docs/application/pdf/fiche bon usage implants cochleaires.pdf.

66. Truy E, Lina G. Implantation cochléaire de l'enfant: technologie, bilan médical et sélection des candidats, réhabilitation. Arch Pediatr, 2003; 10:554-64.

67. Loundon N, Busquet D, Garabedian EN. Implant cochléaire pédiatrique et rééducation orthophonique : Comment adap- ter les pratiques ? Paris: Flammarion Médecine Sciences; 2009. 